\title{
ANALYTIC OVERLOOK OF THE METHODOLOGY OF SYNERGETICS IN POSTNONCLASSICAL SCIENCE
}

\author{
Viktor Yakimtsov ${ }^{1}$ \\ Ukrainian National Forestry University, Ukraine
}

\begin{abstract}
Purpose. This article reveals the main definitions of synergetics and methods that are being used in synergetic research. The differences-characteristics of classical, nonclassical, and postnonclassical science and their schematic illustration are described. There are criteria, by which the main methodological principles of synergetics are being chosen. The reasons that have caused an appearance of synergetics and its methodological apparatus and the framework of this apparatus are considered. The special aspects of nonlinearity of complicated systems, in our opinion, include the economic ones. Methodology. Such foreign and domestic scientists as Wiener N. (2003), Thom R. (1975, 1996), Prigogine I., Stengers I. (1986), Zang V.B. (1999), and Arnold V. (2004) have used methodological apparatus of synergetics in modern science. Methodologically synergetics is open for those new conceptions that are being formed in certain disciplines. Methodological principles of synergetics that cause the "colostral" principles are nonlinearity, nonclosure, and instability. The main principle - the rule of nonlinearity is a contravention of the principle of the super offer in the certain phenomenon (process): the result of adding the impacts on the system is not the adding these impacts' results. The causes' results cannot be added. This means that the result of adding the causes does not equal to the union of causes' results. Results. For the synergy concept, the idea is typical that we see everything at once: the whole and its parts. It combines holism and elementarizm that are continuing each other. Synergetics, in fact, integrates deterministic and probabilistic styles of scientific thinking. Synergetics is the general theory of self-organization. It formulates the general principles of self-organization, valid for all levels of matter. A specific feature of the synergetic approach is to move from the study of simple to complex, from closed to open, from linear to nonlinear, from consideration of equilibrium and near-equilibrium processes to delocalization and instability, to the study of what happens far from equilibrium. Practical implications. The synergetic approach has a great advantage over others. Unlike cybernetics and systems engineering, research which focused on maintaining and preserving the existing state of dynamic equilibrium or order system, synergy, as logical extensions, studying systems in their dynamics and development. Accordingly, if the cybernetics principle operates mainly negative feedback aimed at the preservation of order in the system, synergistic implemented the principle of positive feedback, whereby the changes that occur in the system, not extinguished, and accumulate and amplified. Value/originality. As a result of the interaction of elements of the environment come to coordinated, cooperative behaviour, resulting in spontaneous, a new order, structure or dynamic mode.
\end{abstract}

Key words: synergetics, postnonclassical science, methodology, paradigm, system.

JEL Classification: A13, B41, N10, O10, O30, Q50

\section{Introduction}

During the last decades, a dialectic method of thinking and investigating has given place to brand new - a synergetic approach that is based on the principles of global evolutionism - the combination of sciences about wild-life and inanimate nature, sciences about social development. In the field of economic research, this becomes a brand new level of cognition and provides a brand new decision, which, in its turn, provides the optimization of economic processes' development and the effectiveness of economic growth and, in the last analysis, human well-being.

Effective development of Ukraine's national economy is as of today extremely current and complicated task. Systematic-synergetic aspects and methods of analysis of interrelation between economic processes and phenomena intend to protect the country's economy and increase social well-being. They also will allow considering the problem of effective development of

Corresponding author:

${ }^{1}$ Department of Enterprise Economics, Ukrainian National Forestry University.

E-mail: yakimtsov@nltu.edu.ua 
country's economy not only as a relatively separate system but also as one that has synergetic relations with various subjects of the systematic hierarchy of the country (political, organizational etc.).

There is a real opportunity for understanding and solving the tasks, which are connected to the processes of globalization and internationalization, given that synergetics studies the regularities of appearance, formation, development, and functioning of systems that are self-organized, nonlinear, and open, Economic synergetics, as of today, is on the upswing and ground nonlinear processes of social development.

\section{Results}

2.1. Development of synergetics in the 20th century

From synergetics' perspective, the economy is a selforganized, nonlinear, open, and dissipative system. This system is characterized by multilevel various interactions of its components, the combination of negative and positive feedbacks, interrelation of variability and selection of components, alternation of attractors and homeostasis. These systems are the real structures in open, nonlinear environments, which place the evolutionary processes.

New science is an economic synergetics, which has appeared in the last decades, it forms its own methodology that is based on the laws of general synergetics and determines its categories and definitions.

Based on the above, we have defined the main definitions of synergetics' methodology and methods that are being used in synergetic research.

However, our research would be incomplete without an analysis and estimation of scientists' views on the synergetics as a picture of the world, on synergetics as a research methodology and on synergetics as a science. Such views have originated in different schools of synergetics' scientists and, as it turned out to be, are a force and sense of their investigation and development. They complement the general picture and knowledge base about the synergetics - new postnonclassical research way of investigations of complicated phenomena and objects by dint of complicated modern means and methods etc.

Synergetics as a quite young view of the world has originated due to the necessity of finding adequate, reasonable, calculated and forecasted by science answers to global challenges that civilizational human development makes. Methods of synergetics, as stated above, are based mostly on the regulations of "immortal" science - mathematics.

Methodologically synergetics is open for those new conceptions that are being formed in certain disciplines. Synergetics is inheritable because it falls into interdisciplinary sciences and is based on the methodological framework of mathematics, cybernetics, philosophy etc. In addition, synergetics is self-applicable, because it describes complicated processes and phenomena etc., basing on modern view and cognition of the world. The synergetic methodology is a methodology of interdisciplinary communication and modelling of modern reality, is open for new views, knowledge, methods of research and world cognition the adaptive methodology of establishment and forecast of the most complicated processes and phenomena.

As a rule, in the methodology of synergetics, scientists try to minimize a multivariable set of equations to a small number of significant equations that allows hereafter analysing nonlinear dynamics by significantly shortening (compacting) information.

It can be understood by synergetics:

1. Developmental systems theory (by V.S. Stepin) (2003).

2. Theory of systems that are composed of many subsystems (by H. Haken) (1993, 2003, 2004).

3. Theory of systems that go through the condition of instability and imbalance (by D. Chernavskii) (2004).

Anyway, mathematical methods of synergetics create a multitude that grows and is a base of the methodology of synergetics.

At this date, synergetics is quite fast integrating in the field of liberal sciences, there are approaches of sociosynergetics and evolutionary economics. Methods of synergetics are being used in medicine, pedagogy, linguistics, psychology, history etc.

According to I. Prigogine and I. Stengers's ideas, right before our very eyes, the new worldview is being born a dialogue of human and nature, where human has to realize his role and responsibility in his "community" with the natural laws (Prigogine and Stengers 1986).

For the day, fundamental science pays more and more attention to supercomplex systems, directs its development path into the field of high technologies, economy, forecasts, risks, which forces it to become on the one hand interdisciplinary, on the other hand extremely precise and supercomplex, this science is called postnonclassical.

Postnonclassics as a science approach has originated at the end of XX century in tasks of the description of complicated, evolutionary systems and processes that evolve and self-organize, they can be quite differently interpreted. Thus, the one, who interpret these tasks, becomes a surveillance subject of the process or phenomenon etc. $\mathrm{He}$ is provided not only with surveillance devices, but also with the certain level of knowledge, with culture, psychology, and society (social aspect's impact on his life perception and view of life).

Humanity has travelled the way to such understanding over the last century. From Newton's deterministic physics and Darwin's idea in biology through the principles of relativity theory (A. Einstein) to surveillance facility in quantum physics and hereafter through the modelling of development of the Universe, 
the opening of the role of dynamic chaos and appearance of evolutionary natural science to modelling of historical systems that evolve. Quintessence (centre, core) of these processes is a synergetics. However, it does not mean that its methods can and have to be commonly used; the methods of synergetics do not work where; there is no development of the system (systems).

Postnonclassics is mostly pronounced in tasks of optimal making decisions, ecologic tasks including human's impact on nature, economic tasks of determining human needs considering his psychology and society's possibilities.

Let us briefly describe the differences-characteristics of classical, nonclassical, and postnonclassical science by the scheme of philosopher Stepin, V.S (2003).

Classical paradigm lies in the fact that human raises a question to nature or any object and the object (nature) answers:

The investigation revolves only around the object. The impact of level of researcher's life perception does not count.

Nonclassical paradigm - investigator raises the question to nature and receives an answer from it, but in that answers the object's aspects and the ways that his question's raising are considered.

The principle of the ratio of experiment's result to studying (surveillance) facility is emerging, i.e. there is an impact of surveillance act on the system (object), which cannot be neglected. It can be schematically illustrated in this way:

An express condition of nonclassical paradigm is the consideration of ways and methods of posing the questions and object's aspects.

Postnonclassical paradigm lies in the fact that the investigator raises the question to nature, which gives an answer, but now the answer depends also on quality and characteristics of the investigator, object, ways of investigation, methods and level of understanding of the subject-investigator.

As of today, such an approach is considered the newest, objective, and giving ample opportunities for studying the complicated phenomena and processes that are the object of synergetics' studying.

\subsection{Methodological principles of synergetics}

The modelling of complicated systems, which evolve, not only of natural and of technical but also of liberal and economic, becomes mainstream and interdisciplinary direction of science in XXI century. That is why it is necessary to distinctly present the principles and methods (technologies) of approaches to such systems' modelling.

The main methodological principles of synergetics are being chosen by several criteria:

1. The principles of the methodology of synergetics can and mostly have to be in such dependence that they could be expressed one in terms of another. For example, the definition of "gene" cannot be defined without such definition as "organism", which part it is.

2. Systems of complicated phenomena and processes can be modelled and built in various coordinate systems of various measurements. Then the problem of conversion of such systems from one coordinate system to another appears.

3. The number of principles has to be enough, but not too big. A person, who uses them, will not have an opportunity to simultaneously keep track of their maintenance in real modelling.

4. Systemacity, systematic approach to the study of complicated processes, phenomena etc. This is an express condition (principle) of the methodology of synergetic investigations in any field of their use as of today.

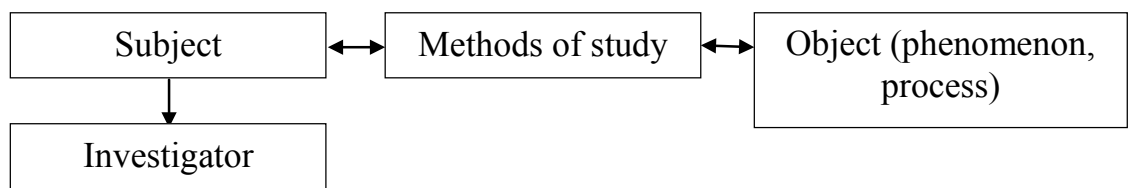

Fig. 1. Schematic illustration of classical paradigm's core

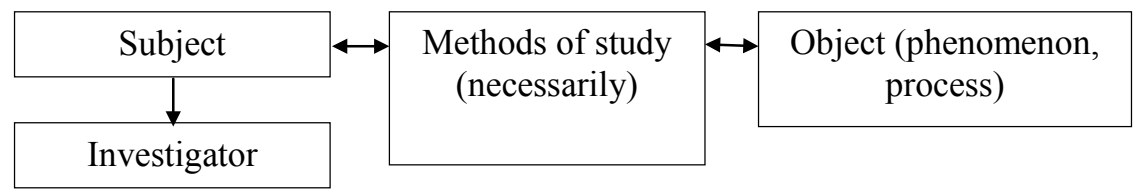

Fig. 2. Schematic representation of nonclassical paradigm's core

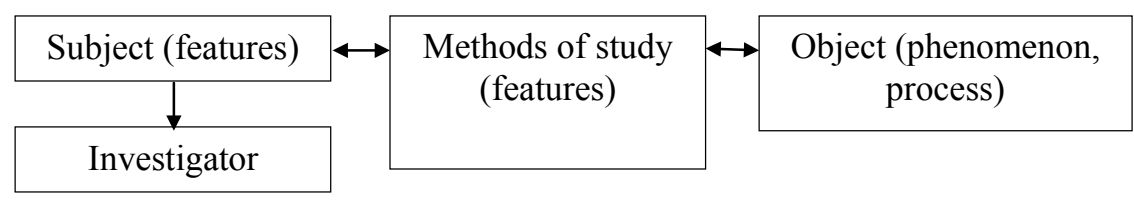

Fig. 3. Schematic representation of postnonclassical paradigm's core 
Professor Budanov, V.G. (2007) has studied the methodological principles of synergetics from a critical point of view of their really practical use in economic tasks of modernity.

Any evolutionary process is presented by a range of changes in conventional conditions of order and chaos in systems that are linked by phases of transition to chaos (system's destruction) and way out of chaos (self-organization). Only one of above said stages we call a homeostasis of the system. This is a stage of the table existence of complicated system as it was called Existence by prof. Budanov (2007). It, as a rule, is the longest and has stable characteristics, the rest of stages of existence of conditions of existence of complicated systems, phenomena, processes are called the colostral stages and crisis stages. Such a classification is somewhat conventional because even in steady state condition of system's existence, there is an element of chaos and the element of order may be found in chaos (crisis). The problem is in the degree of their blend, i.e. correspondence of one small part to another. Nature, as a rule, evolves by "baby" steps, so the condition of Existence is inherent to it. The principles of this condition are: 1) homeostatic and 2) hierarchical pattern. They characterize the phase of order, the stability of system's functioning, clarity and description's simplicity. For example, in nature, it is a day-night biorhythm, the circle of seasons etc.

Prof. Budanov has distinguished also other five principles of the colostral stage: 1) nonlinearity; 2) imbalance (instability); 3) nonclosure; 4) dynamic hierarchical pattern; 5) surveillance (observance) (2007).

These principles characterize the transformation stage, system upgrade, passing successive steps by this system by old order's destruction and chaos of change of alternatives and eventually the inception of the new order.

Herewith first three principles are the principles of the methodology of synergetics that give birth to the colostral stage of the new system of order. And the last two of them are constructive, they provide the new system with its compilation of corresponding details and constructions (characteristics-aspects) and also give investigators an opportunity to understand, observe and describe "the new order" and they give him its coexistence with the environment.

Let us have a closer look at above-said principles and estimate them from a perspective of using the economic systems in synergetic methodology.

Ultrastability of the system is a phenomenon, which has to keep the system's functioning within bounds - limits that allow it to have a sense of purpose (goal). According to N. Wiener, any system has a purpose (goal) of its existence, according to which the systems' motion is being corrected (2003). Such a correction is being provided by corresponding signals, characteristics, features, and aspects, which hold any negative impact from outside under. For example, this is exactly how homeothermal creatures behave in nature when for survival as a goal they maintain the stable body temperature despite any temperature variations in the environment.

The purpose (goal) - the program of system's behaviour in the condition of homeostasis (ultrastability) is called an attractor in synergetics. In space of system's conditions, the attractor is a certain variety of characteristics of the phenomenon, process etc. which in due course the closest system's conditions that are being drawn to.

Area (field) of attractor's attraction is called his basin. It is worthy of note, that attractors exist only in open dissipative systems, i.e. systems that dissipate the energy, material and information and describe the final behaviour (condition) of the system, which usually is simpler than transient process.

Examples of simple attractors in mechanics are a pendulum that attenuates in the lowest point of motion or a ball at the bottom of the hole. These are the attractors of equilibrium.

Nevertheless, more complicated attractors are possible too, for example, the flight of an eagle in the air blasts, the air, on the one hand, moves from the ground and on the other hand - downwind. If there is no such motion, then the eagle will not be able to hover (fly). Such structures that exist only until they contain the additional flows of energy or material are called dissipative (those, which dissipate the energy) - are far from equilibrium and stability.

Living systems of existence are exactly such systemsstructures - they die without a constant inflow (flushing) of energy or material through them, i.e. without the metabolism. This principle unites the ideas of cybernetics, system analysis, and eventually - the synergetics.

One more important principle is a hierarchical pattern of the systems-structures. The main hierarchy's matter in nature is the fact that constitutive nature is the highest level in relation to lower ones that are a structureless element of chaos, i.e. just a construction material.

For example, the hierarchical system "society" is the highest level, which consists of the lowest - people that form this society; atoms of material consist of cores, electrons etc. These are words, phrases, texts etc. in language; these are understanding, views, ideologies etc. in the world of ideas (science). These levels are hierarchical structures (ladders) that exist everywhere around us.

Always, when elements are being united in structures, they pass them a part of their characteristics, which become the characteristics of the system itself. Such system's characteristics are called "the order parameters" and their functioning nature is called the principles of submission, i.e. the variation of a parameter of the 
lowest order, by giving its characteristics to the general system, submits the generalized characteristics of the system (structure) of the highest order.

The phenomenon of their specific, i.e. mutually agreed coexistence is called self-organization. It is worthy to lay emphasis on circular causality in phenomena of selforganization, interdependence and interconditionality of the behaviour of elements of two neighbouring orders. For example, in the stream (flashy flow), there is a water motion (main one) that sucks the small substances (sand, leaves etc.) in and forces them to move along with the water. These substances, in their turn, give their characteristics, in this case - pollutions, to the water.

An important characteristic of such hierarchical systems is the impossibility of full reduction (junction of its components) - a reduction of characteristics of structures of more complicated orders to the language (motion, features) of structures of more simple (low) orders of the system.

A specific role in systems' hierarchy is time because the synergetic principle of submission if described and defined by $\mathrm{H}$. Haken exactly in time - temporary system's hierarchy (1993, 2003, 2004). Such closest levels in systems' investigation are micro, macro and mega levels.

Above said principles of existence need their supplement with colostral principles, which are the driving force of evolution.

Methodological principles of synergetics that cause the "colostral" principles are nonlinearity, nonclosure, and instability.

Main principle - the rule of nonlinearity is a contravention of the principle of the super offer in the certain phenomenon (process): the result of adding the impacts on the system is not the adding these impacts' results. The causes' results cannot be added. This means that the result of adding the causes does not equal to the union of causes' results. Customary borshch can be an example, it consists of $n$-number of ingredients and in the same time, it is not a simple union of its components, but it is the new quality of these components under the influence of heat treatment of these ingredients.

The principle of super offer does not extend to nonlinear systems when two factors' effect on the situation causes an effect, which has nothing to do with the results of the certain effect of each factor. The development in nonlinear systems is by nonlinear laws that lead to the multivariance of ways of selecting and of alternatives of exit from the state of imbalance and instability.

In nonlinear systems, the processes, phenomena etc. can be of deeply threshold nature, when there is their slip-and-stick transformation to another feature during the gradual change of external conditions. Herewith, the old structures are being damaged, sometimes there is their full destruction and they transform to qualitatively new structures.
Unbalanced, open nonlinear systems constantly create and maintain the inhomogeneity in the environment. There some unexpected consequences can appear between the environment and the system, because there the relations of positive feedback appear, which strengthen system's equilibrium deviation.

In the processes of analysis and synthesis of nonlinear systems' components that self-organize, the resulting deduction (the answer) can be determined not by simple adding of each component, but by synergetic method - generalized synthesizing impact not only of each component separately but also of their generalized effect in a greater degree.

Such an approach in economic research is extremely necessary, but unfortunately, as of today, it is not commonly used even in scientific research. In actual fact, it is the newest approach to study the economic processes, which is debatable, but extremely necessary for modern insight and generalization of processes of society's economic growth.

\subsection{Prospects for synergetics}

Today, in conditions of accelerated and instable development of the world and economic processes, the synergetics and its methodological apparatus have, in our opinion, the defining role. This is an attempt to realize, describe, solve, and forecast the principles of evolution of economic systems, phenomena, processes etc., to consider the causes of economic recoveries and recessions.

The overwhelming majority of macroeconomic models that were developed and described earlier and as of today are the axioms or close to them for one economic process or the other, contain assumptions and are based on the postulate that the economy is a closedloop system. However, it means that the processes that occur in the closed-loop systems according to the second law of thermodynamics are being drawn to the equilibrium and in due course, these processes attenuate and stop. Therefore, supposing that economic processes are being described by the closed-loop system, it is logical that these attenuating processes will lead to their edge state - the equilibrium.

However, the economy and its processes are the closed-loop system that falls under influence of various external and internal factors. Professor V. Nusratulin (2006) has proved that the economy is instable and closed-loop by its core and nature.

The special aspects of nonlinearity of complicated systems, which, in our opinion, contain the economic ones, lie in these facts:

- nonlinearity causes indignations and agitations of the systems (fluctuations) and can strengthen or reduce them. For example, the investment in one economic process or the other, even quite insignificant increase of financial assets (financial investment volume) causes significantly bigger GDP growth; 
- nonlinearity causes threshold sensitivity to the changes. For example, in the economy, the demand decreases when the production price raises and conversely;

- nonlinearity provides the suddenness of changes in course of processes in the system because many various factors have a significant impact on it;

- nonlinearity's special aspect is the fact that under a certain number of changes in the environment, the transformation to another feature may not happen. True and reverse thesis - qualitative change of the environment causes the appearance of new opportunities - system's components, which will lead to the change of the system itself.

The impossibility to neglect the external and internal conditions' impact on the system is a specific principle of the methodology of synergetics that is according to nonclosure (openness) of the system.

All living systems, as well as society, are open systems; they consume the material and energy. It is the openness that allows such systems to evolve from simple to complicated one, to open a program of organism's growth from cell-embryo to highly organized grown organism. This means that hierarchical level might develop and be complicated only during the exchange of material, energy, and information with other levels.

Inanimate nature, dissipation (system's transformation of energy, which is arriving at the system to thermal energy) can also lead to regulation of the structures. For example, the evolution of the solar system. The synergetics has originated of the description of such systems in chemistry, physics and theory of laser. The most interesting the homeostatic structures are the structures that are not in equilibrium with the environment, in other words, such systems, which do not have maximally possible entropy.

Of course, any process, when the system transforms from one condition to another, occurs that way that it is impossible for this process to be in a reversed direction for the system to experience identic intermediate states and in such event for the surrounding bodies not to experience any changes. Herewith the energy dissipation will certainly occur at the expense of emission, abrasion etc. Therefore, almost all processes (phenomena) in nature are inconvertible; a part of the energy is being lost in them. The concept entropy is being introduced for characterizing the energy dissipation.

Therefore, the entropy is a function of system's condition, when very insignificant changes in reversible process are equal to the ratio of the very small amount of heat that was added in this process to the temperature, which was there when this heat was added.

The entropy can be constant only in closed-loop systems, i.e. such systems, where there is no energy exchange with external conditions (bodies). The word "entropy" in Greek (entropia) means the turn, transformation. This concept was introduced to determine the measure of energy dissipation during the reversible processes in thermodynamics; this is a measure of the probability of any macroscopic state in physics, the measure of indeterminacy of certain research that can have various results - in information theory. Entropy as a sort of "measure of chaos degree" in systems is an extremely important characteristic in synergetic research about complicated open systems.

The majority of objects that we are interested in and which are being investigated in this work are economic structures and processes, ecologic and natural phenomena, enterprises, social phenomena etc., are the open systems, instable, imbalanced, open and are being managed by nonlinear laws.

The principle of nonclosure (openness) of the systems by hierarchical levels lays emphasis on two extremely important circumstances:

1. Processes' self-organization becomes possible - the openness of processes, structures, and systems of the macro level will lead to the possibility of the existence of same phenomena on the micro level.

2. Analogous phenomena might also occur at the level of structures (systems) of the macro level and the mega level of managerial parameters of systems that change.

During the transformation from one system's condition to another, the system becomes in a mandatory manner open in point of imbalance (instability).

One more methodological principle in research about synergetic systems is an instability, disequilibrium, imbalance. The system is instable if there are conditions that cause any small variations of its parameters, which change the system's condition. Such systems' characteristic was considered their disadvantage for a long time and bifurcation points - points of system's transformation to another condition were considered the points that manage the systems.

The significance of bifurcation points also lies in the fact that influence on the system's behaviour, its further development and condition can be made only in them. It is also worthy of consideration that such circumstances as the unpredictability of bifurcation points' impact on the behaviour and hereafter on the development of systems' condition.

R. Thom (1975, 1996) and V. Arnold's (2004) catastrophe theory is sometimes called the bifurcation theory because it is able to forecast the process, which can occur in the future.

There are two cross-functional pre-crisis symptoms of system's behaviour, i.e. two "premonitory symptoms" of catastrophes. The first one is called "the lull before the storm" or the pre-crisis slowdown characteristic rhythms of the system. The second one is an increase of noise fluctuations in the system near the bifurcation point, i.e. increase of chaotic variation of system's characteristics from their average values. 


\section{Discussion and conclusions}

Synergetics can and even has to study such systems by constructive principles. Constructive principles are the principles that give the investigator the opportunities to unite, coordinate and accumulate the principles of synergetics.

The development of interdisciplinary approaches turned out to be very close in form and substance to teaching or scientific popularization. In both cases, it is necessary to comprehend the way passed by researchers, highlight it in the key ideas and results, most important "for the uninitiated", and also ruthlessly throw out many details "dear to the author." This path may present unexpected generalization and a new vision of problems to be solved.

Nonlinear dynamics (synergetics) offers a basic model, new concepts and techniques, that can be applied in this situation, and that cannot be. They can be the basis of the building of a new nonlinear cognitive paradigm and may remain separate findings in various disciplines.

Synergetics seems us to be no dogma or even no guide to action, but a way of looking at the problem, which is sometimes very useful, in fact. Although, of course, we cannot discount the form, fashion, and charm of the people involved with the synergy.

\section{References:}

Arnold, V.I. (2004) Theory of catastrophes (in Russian), 4th ed. Moscow, Editorial-URSS.

Budanov, V.G. (2007) Methodology of synergetics in the post-non-classical science and education, Institute of Philosophy. M.: LCI Publising house, $240 \mathrm{p}$.

Chernavskii, D.S. (2004) Synergetics and Information. Dynamical Theory of Information. URSS.

Haken, H. (2004) Synergetics: Introduction and Advanced Topics (Springer, Berlin, Heidelberg)

Haken, H. (1993) Advanced Synergetics: Instability Hierarchies of Self-Organizing Systems and Devices. New York: Springer-Verlag, $424 \mathrm{p}$.

Haken, H. (2003) Secretes of Nature. Synergetics: the Learning about the Interaction, $320 \mathrm{p}$.

Limited (2006) Chichester, 1983. Regular and Chaotic Dynamics, $136 \mathrm{p}$.

Wiener N. (2003) The Creator and the Future, 732 p.

Nusratullin V.K. (2006) Nonequilibrium economy. 2nd prod. additional M: Satellite company, 482 p.

Prigogine, I. Stengers, I. (1986) Order out of Chaos. Man's new dialogue with nature, $432 \mathrm{p}$.

Stepin, V.S. (2003) Self-developing systems and postnonclassical rationality. Moscow.

Thom, R. (1975) Structural Stability and Morphogenesis: An Outline of a General Theory of Models. London.

Thom, R. (1996) Logos et Théorie des Catastrophes: à partir de l'oeuvre de René Thom, ed. Jean Petitot. Colloque de Cerisy-la-Salle 1982 (Patiño, Geneva 1996).

Zang, Wei Bin (1999) Synergetic Economics. Time and Locusts in the Nonlinear Economic Theory / V. Zang, M. Ostrovsky (translated from English) - Moscow: Mir, 366 p. 